\title{
TRANSIT-TIME FLOWMETRIC EVALUATION IN CORONARY ARTERY BYPASS GRAFTING
}

\author{
Veselin Petrov \\ Department of Cardiovascular Surgery and Angiology, Faculty of Medicine, \\ Medical University of Varna
}

The problem discussed in the article "Transit-Time Flowmetric Evaluation of Coronary Artery Bypass Graft Flow after Off- and On-Pump Myocardial Revascularization" by V. Kornovski et al. is of huge clinical importance. The routine use of a graft flow verification tool is mandatory for the surgeon in order to remove any doubts about the anastomotic patency at the end of coronary artery bypass surgery. This can eliminate the possibility of a technical error during the intervention and thus, to minimize the possibility of developing a serious perioperative complications. The results of the author are encouraging. There is an important fact: the good quality of the anastomoses in both operative methods - off-pump and on-pump coronary artery bypass is a very persuasive evidence that this diagnostic tool is of great practical value.

If we consider that reoperations for graft failure are time- and money-consuming, together with additional work in the operating room for the heart team, the meaning of this device is of even greater importance. In experienced hands this method is quick and reliable, and provides the surgeon with evidence of patency of the grafts postoperatively, which is an important factor in the early postoperative period, especially when some hemodynamic or other problems occur.

Citation: "Our results indicated that both clinical effectivity and safety of OPCAB surgery can be compared to those of the ONCAB one in terms of postoperative graft patency, coronary graft blood flow and PI values." 
